\title{
Counteradaptation after exposure to displaced visual direction*
}

\author{
HANS WALLACH and DAVID HUNTINGTON \\ Swarthmore College, Swarthmore, Pennsyliania 19081
}

\begin{abstract}
Counteradaptation, previously demonstrated in connection with adaptation in distance perception, was obtained after exposure to displaced visual direction. When $\mathrm{S}$ adapted to a laterally displacing wedge prism by walking during the exposure period, there was not only a change in the perceived visual direction, but also a change in the proprioceptively perceived walking direction. When $S$ adapts to lateral displacement of the visual direction by looking at his stationary or his moving arm, visual adaptation is obtained in the latter, but not in the former. case (Held \& Hein, 1958). We obtained a change in the proprioceptively perceived position of the arm when it was stationary during the exposure period. a condition which had not yielded visual adaptation, and a much smaller, not significant, change in the felt position in the case of the actively moved arm. In the present experiments, changes in proprioceptively perceived direction or position amounted to counteradaptation.
\end{abstract}

At this time, it seems useful to distinguish three kinds of perceptual adaptation: adaptation in the perception of shape in the frontal plane, adaptation consisting of the alteration of a constancy. and adaptation in the modification of cue evaluation. We are concerned here only with the latter. A modification of cue evaluation consists of a quantitative change in the relation of a cue to the perceptual property for which the cue provides the sensory information. Examples of such properties are distance perceived on the basis of accommodation and convergence, stereoscopic depth, visual direction, and the perception of verticality or slant. Devices that change accommodation and convergence, alter interocular distance, change visual direction by means of wedge prisms, or slant the visual field alter stimulation so that it misrepresents the objective conditions and nonveridical perception results. Eventually, a compensatory change in the perceptual process partially or totally corrects for the faulty sensory input-adaptation to the device takes place, with the result that veridicality of perception is partly or entirely restored. Such a compensating process change can, of course, take place only if veridical information about the pertinent objective conditions reaches the nervous system. Such information may be in a different modality from the altered stimulation, or it may be in the same modality provided it is not affected by the device to which $\mathbf{S}$ adapts. This veridical information must be contained in a sensory cue that pertains to the same perceptual property produced by the altered stimulation. In the case of adaptation in distance and in depth perception, sources of veridical information have been investigated (Wallach, Frey, \& Bode, 1972, and Wallach, Moore, \& Davidson, 1963, respectively). Where adaptation in distance perception is concerned, these

*This work was supported by Grant $25.000 \mathrm{X}$ from the National Science Foundation to Swarthmore College. Hans Wallach. principal investigator. We are most grateful to Charles $\mathrm{S}$. Harris for his critique of the first version of this article and for his many suggestions for its improvement. sources consist of cues for distance other than accommodation and convergence, the altered cues. It seems, then, that in the kind of adaptation where cue evaluation is modified, we have an altered cue and one or more veridical cues all related to the same perceptual property. We believe that this is also true of adaptation to displaced visual direction and to visual tilt. We call different cues pertaining to the same perceptual property "paired cues." Under ordinary circumstances, each member of a cue pair operating by itself should produce the same value of the perceptual property which they both determine. ${ }^{1}$ When one is altered by a device while the two perceptual processes resulting from the two cues still take their normal course, different values of the common property should result. Eventually, a compensating modification in one of the perceptual processes may take place such that the outcome of the two processes will again be in agreement. This is the process assimilation hypothesis first proposed by Wallach and Karsh (1965) and discussed in detail by Wallach (1968). According to this hypothesis, process modification serves to diminish the initial difference in the outcome of the two perceptual processes that results when one of a pair of cues is altered by a device.

It is an essential feature of this hypothesis that such process modification does not necessarily amount to a compensation for the effect of the device and hence to adaptation. Process modification will amount to adaptation only when it changes the process that is produced by the altered cue. If the process that is caused by the veridical cue is modified in order that the agreement in the ultimate outcome of stimulation by the cue pair be restored, no compensation for the device occurs, and veridicality is not enhanced. Instead, a process is so modified that perception based on a veridical cue becomes nonveridical. It is a consequence of the process assimilation hypothesis that such a change in perception toward nonveridicality occasionally occurs when $S$ is exposed to a cue-altering device. Wallach and Frey (1972) have introduced the term "counteradaptation" to refer to process modification of this sort. 

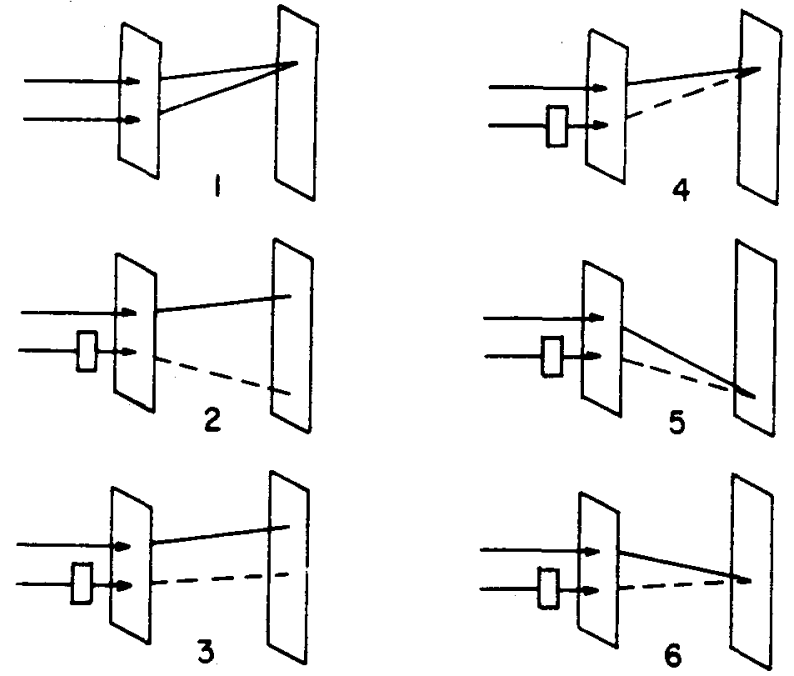

Fig. 1. Various forms of process assimilation.

Since the possible occurrence of counteradaptation is a consequence of the process assimilation hypothesis, demonstrating counteradaptation after exposure to a cue-altering device serves to confirm this hypothesis. But this is not the only reason why one might want to test for counteradaptation. Finding that counteradaptation develops along with a certain kind of adaptation or instead of it may solve certain problems that arise in connection with adaptation, such as failure of adaptation to develop where it would be expected to occur or an adaptation falling short of complete compensation-for the effect of the cue-altering device. Figure 1 illustrates the various forms process assimilation may take. In the six sketches, the smaller vertical planes represent stimulation and the two horizontal lines, a cue pair impinging on a sensory surface. A point in the larger vertical plane represents the outcome of a perceptual process, experience or performance (such as judgment or pointing) or an intervening variable (such as registered distance). The lines connecting the planes represent the course the perceptual processes take. Sketch 1 shows the processes caused by the pair of cues producing the same value of their common perceptual property, the two process lines ending at the same point in the larger plane. This is what happens under ordinary circumstances. In Sketch 2, a device is introduced that causes a cue alteration so that it produces a different perceptual process with an outcome different from the process caused by the veridical cue. In Sketch 4 , full process assimilation has occurred, with the process corresponding to the altered cue having undergone all the change; this is the case of complete adaptation, i.e., the outcome is the same as before the device was introduced (Sketch 1). Sketch 3 depicts partial adaptation. Full process assimilation has occurred also in Sketch 5 , but here the process caused by the veridical cue has done all the changing and the value toward which the processes have assimilated is nonveridical. This is complete counteradaptation. It is a possible explanation where an expected adaptation fails to develop. Finally, Sketch 6 depicts the case of complete process assimilation due to simultaneous adaptation and counteradaptation. It can account for the case where adaptation develops but stops short of being complete, because counteradaptation has contributed to process assimilation becoming complete. ${ }^{2}$

So far, few examples of counteradaptation are known, because specific testing is required to show that it occurred. Wallach and Frey (1972) caused a change in visual distance perception to take place as counteradaptation. They selected a condition where only one kind of cue served as veridical input in adaptation to glasses that changed accommodation and convergence with which objects were viewed. They transformed this condition in such a way that, while the cue discrepancy remained the same as before, the formerly veridical cue became nonveridical, and that accommodation and convergence now represented the objective facts correctly. Since the course that process assimilation takes supposedly depends only on the cue discrepancy when a simple cue pair is in operation, Wallach and Frey obtained the same process change as before, except that, under the new conditions, it amounted to counteradaptation. Wallach and Smith (1972) demonstrated proprioceptive changes amounting to counteradaptation along with adaptation in visual distance perception after $\mathbf{S}$ observed his moving hands through glasses that changed accommodation and convergence. The present report is on two experiments in which counteradaptation in proprioception was obtained after exposure to displaced visual direction.

\section{EXPERIMENT I}

A condition often used to obtain rapid adaptation to displaced visual direction consists of having $S$ walk with a wedge prism in front of one or both eyes. Here, a cue discrepancy exists between the proprioceptively and the visually given walking direction, the former veridical and the latter changed by $11 \mathrm{deg}$ by a 20 diopter prism. The visual walking direction is probably given primarily by the focus of expansion of the visual scene (Gibson, 1950 , p. 128). The direction of this focus normally coincides with the walking direction, but when a wedge prism is worn and, together with the whole visual field, the focus of expansion is laterally displaced, a discrepancy between the visual and the proprioceptive walking direction results. A process assimilation that responds to this discrepancy can here either come about by a change in the relation between the cues for visual direction and the resulting visually perceived direction, a change that would compensate for the effect of the prism, or it can come about by a change in the evaluation of proprioceptive cues for the walking direction in a nonveridical direction. The former would 
amount to adaptation, the latter to counteradaptation. We expected that. if a change in the evaluation of proprioceptive cues developed, it would manifest itself in a discrepancy between an intended walking direction and the direction in which $S$ actually walked. We probed for such a change in the actual walking direction with two tests. In one, $\mathrm{S}$ was asked to walk straight ahead in a totally dark room, and in the other test, also in the dark and after the prism had been taken off, he was asked to walk in the direction of a target point that had briefly lit up before he started to walk. In the latter test, an error in the walking direction would not only reflect a proprioceptive change, but also a change in the visual direction of the target point. But a third test that measured visual adaptation, as such, made it possible to demonstrate a proprioceptive change as the difference between the walking error and the measured visual adaptation. In the test for visual direction, $\mathrm{S}$ had the task of changing the position of a luminous target point in the horizontal dimension until it appeared straight ahead of him (visual forward-direction test).

What changes due to the exposure conditions can these tests be expected to turn up? If the prism causes an average shift by $11 \mathrm{deg}$ of all visual directions, for instance, to the right, the focus of the field expansion caused by S's forward motion will lie also $11 \mathrm{deg}$ to the right of the direction in which $\mathrm{S}$ walks. If $\mathrm{S}$ were conscious of the focus of field expansion, which he is not, he would see it lie 11 deg to the right of his walking direction, provided he could experience this walking direction correctly, which he probably cannot. ${ }^{3}$ Whether it is represented in conscious experience or not, this is the initial perceptual result of the cue discrepancy produced by the prism. If now process assimilation becomes effective, it diminishes this difference in the outcome of the two processes, with this difference, it will be remembered, being the normal result of the given discrepancy between the visual and the proprioceptive cues. Process assimilation may amount to a shift of the perceived visual directions to the left and to a shift of the proprioceptively perceived walking direction to the right of the actual walking direction. The former is, of course, the familiar visual adaptation where a change in the relation between the given and the perceived visual direction compensates for the effect of the prism, enabling $S$, for instance, to point at a visual target with less of the error than the prismatic displacement of the target's direction would warrant. If due to process assimilation, the proprioceptively perceived walking direction has come to be to the right of the actual walking direction, $\mathrm{S}$, intending to walk in a certain direction, will actually walk a path to the left of the intended direction, and this will, of course, be counteradaptation. In the test in which $S$ is asked to walk straight ahead, his actual walking direction will be to the left of forward. and when he has to walk in the direction of a previously exposed target point. it will be to the left of its visually perceived direction. The latter. because of visual adaptation, will, in turn, be to the left of the objective target direction, if the test is made without the prism. The effects of adaptation and counteradaptation will add, each having served in its way to compensate for the cue discrepancy caused by the prism.

\section{Procedure}

During the exposure period. S wore welder's goggles with a 20 diopter wedge prism mounted in front of the right eye. His left eye was covered with a patch. For six Ss, the base of the prism was to the left, so that the prism caused a displacement of the visual direction to the right, and for the other six, the base was to the right, causing a displacement of the visual direction to the left. The prism was covered with Wratten Filter 58 to eliminate color fringes and the attendant loss of acuity. During the exposure period, which lasted $20 \mathrm{~min}, \mathrm{E}$ accompanied $\mathrm{S}$ on a walk through the building along wide hallways interspersed with climbing stairs and entering rooms. S was instructed not to look at any part of his body or to touch objects in his path.

All tests were made without prism in a darkened room. Here, too, S's left eye was patched. For the visual forward direction test, the apparatus described by Wallach, Kravitz, and Lindauer (1963) was used, with one change-enabling $S$ to change the target direction himself. $S$ stood with his teeth in a mold, facing a horizontal frontal-parallel track. $58 \mathrm{~cm}$ from his eyes. On it rode a sliding fixture with a dimly lit $2-\mathrm{V}$ bulb at eye level. With the help of two strings, one for each hand, $S$ could move the bulb along the track. He was instructed to move the bulb until it appeared "directly in front of you, straight ahead." After S was satisfied with his setting, he was asked to close his eyes and $E$ read the position of the bulb from a scale in back of the track. A test consisted of two such settings, one starting from the extreme left and the other from the extreme right; their mean became S's test score.

In the walking-straight-ahead test, $\mathrm{S}$ was put in a fixed starting position. with the orientation of his body controlled by a board on the floor against which $S$ had to back his heels, his feet spread $40 \mathrm{~cm}$ apart. He was asked to walk straight ahead until he reached a string that was stretched across the room $2 \mathrm{~m}$ from his starting position and to stop there. S's position was measured. and from it his walking direction was computed. This forward walking was repeated five times, and the mean of the five measurements became the test score.

A similar arrangement was used for the walking-toward-a-target test. A bulb at $S$ 's eye level was located $2.5 \mathrm{~m}$ from S's fived starting position. $50 \mathrm{~cm}$ beyond the string barrier. The bulb was dimly lit for 2 or $3 \mathrm{sec}$, and, when it was extinguished, $S$ was instructed to "walk to where you saw the light." $S$ stopped at the string, and his position was measured. This procedure also was repeated five times in each test.

All three tests were given before and after the exposure period. The test order was varied from $S$ to $S$. but was. for a given $S$, the same before and after the exposure period. Twelve Ss were used to fill the six test orders twice.

\section{Results}

The mean differences between the walking directions before and after the exposure period and the corresponding angular differences in the target setting in the visual forward-direction test are given in Table 1 . When the difference scores produced by exposures to right displacement and to left displacement were combined. the visual effect amounted to $3.1 \mathrm{deg}$, which is in line with previous results on visual adaptation. ${ }^{4}$ The 
Table 1

Mean Differences Between Directions Measured by the Pre- and Postexposure Tests in Degrees

\begin{tabular}{llll}
\hline Test & $\begin{array}{c}\text { Exposure } \\
\text { Displace- } \\
\text { ment }\end{array}$ & $\begin{array}{c}\text { Mean } \\
\text { Difference } \\
\text { Scores }\end{array}$ & \multicolumn{1}{c}{$\mathrm{p}^{*}$} \\
\hline Forward & Right & 5.0 Left & $<.05$ \\
Walking & Left & 4.0 Right & $<.05$ \\
& Combined & 4.5 & $<.01$ \\
Visual & Right & 3.6 Left & $<.05$ \\
Forward & Left & 2.7 Right & $<.1$ \\
Direction & Combined & 3.1 & $<.01$ \\
Walking & Right & 7.4 Left & $<.001$ \\
Toward & Left & 5.3 Right & $<.01$ \\
Target & Combined & 6.4 & $<.001$ \\
\hline
\end{tabular}

*All probabilities are for two-tailed tests.

effect measured by the forward-walking test amounted to $4.5 \mathrm{deg}$. and this is counteradaptation in proprioception. The two effects are measured in combination in the walking-toward-target test, which showed a mean difference of $6.4 \mathrm{deg}$. There was no effect of test order, and the slightly smaller effects obtained with exposure to left displacement ${ }^{5}$ were not significantly different either. The experiment, thus, yielded two demonstrations of counteradaptation in proprioception, the result of the forward-walking test and the difference of the results for the walking-toward-target test and the visual forward-direction test. This difference was significant $(\mathrm{p}<.02)$.

\section{EXPERIMENT II}

With proprioceptive counteradaptation after exposure to displaced visual direction thus demonstrated, it seemed worthwhile to examine a case where visual adaptation to wedge prisms has failed to occur. As stated, the process assimilation hypothesis suggests that a way to account for failure of an expected adaptation to occur is to demonstrate that counteradaptation develops instead. The experiment of Held and Hein (1958) and Held and Bossom (1961) are instances. In both cases, a visual adaptation to wedge prisms was obtained when Ss moved and did not occur when Ss remained passive during the exposure period. We believe, however, that the reasons for obtaining these results were different in the two experiments. In the experiment by Held and Bossom, Ss walked during the exposure period or were pushed on a wheelchair over the same path. We explain the fact that adaptation failed to develop in the latter case by the absence of a cue discrepancy. Being leisurely moved on a wheelchair probably produced no proprioceptive stimulation carrying information about S's direction of motion. ${ }^{6}$ Held and Hein, on the other hand, had S during the exposure period look at his arm and then tested for adaptation. When $\mathrm{S}$ moved his arm back and forth. an effect amounting to about $35 \%$ compensation for the visual displacement (a shift of $3.8 \mathrm{deg}$ ) was obtained. But when the inspected arm remained stationary, no adaptation was measurable. In the latter case, the exposure condition does provide a cue discrepancy, the difference between the visual and the proprioceptive location of the arm. The failure of adaptation to develop when the arm remains stationary may well be attributable to counteradaptation in proprioception, a change in the felt location of the arm. We tested for a change in the felt location of the inspected arm by having the blindfolded $S$ point with his other hand to an easily identified part of the inspected arm, first before and then again after the exposure period. If counteradaptation was responsible for Held and Hein's result, we would expect to find a change in the felt location of the inspected arm when it had remained stationary during the exposure period and a smaller change or none after observation of the moving arm. There were, then, two experiments, one with the arm stationary during the exposure period and the other with the arm continuously moving.

\section{Procedure}

Throughout the experiments, $S$ sat at a table with his left arm resting or moving on the black table top. with the hand closed in a fist, his head positioned by a teeth mold. During the exposure periods. $S$ wore the same goggles as in Experiment I. In the stationary-exposure condition. the arm was so placed by $E$ that the knuckle of the middle finger was located in S's median plane $44 \mathrm{~cm}$ from his eyes, and $S$ looked at his motionless fist for $10 \mathrm{~min}$, with his right arm out of sight. In the moving-arm condition. S's elbow was in the same position on the table as in the stationary condition. but $S$ was instructed to move his lower arm and hand back and forth between two marks on the table. $28 \mathrm{~cm}$ apart, while looking at his fist. This exposure period also lasted $10 \mathrm{~min}$; this time, however, it was broken midway by a $40-\sec$ rest period, during which $S$ sat with eves closed and arm stationary.

In the tests, the blindfolded $\mathrm{S}$ had to point with the index finger of his right hand at the knuckle of the middle finger of his left fist, the one that had been viewed during the exposure period. S's pointing finger, however. did not touch his left hand. but, instead, made contact with a Plexiglas plate that, for the test, was swung into position just above the left fist. When, before the preexposure test, $\mathrm{S}$ was instructed in this task, he was allowed actually to touch the knuckle a few times with eyes open "to get the feel of it." Then S bit into the mold, was blindfolded, and the Plexiglas plate was swung into place. $E$ then moved S's arm into one of the two test positions so that the knuckle was directly beneath one of two marks on the transparent plate. These locating marks were $8.5 \mathrm{~cm}$ to the left and $8.5 \mathrm{~cm}$ to the right of S's median plane. To make a pointing. $S$ brought his right hand into position above his left fist so that his index finger pointed to the spot where he felt the knuckle to be and then lowered his hand until the pointing finger touched the plate. E marked the position of S's finger on the plate, and S dropped his hand to the side. This pointing procedure was repeated two more times. Then $E$ moved S's left arm and hand in the other pointing position. and three more pointings were made. Finally. E moved S's left fist into the exposure position midway between the two test positions. the plate was swung out of the way, and the blindfold was exchanged for the goggles: the exposure period began. The tests were repeated after the exposure period. 
The same $16 \mathrm{Ss}$ participated in both versions of the experiment. with an interval of at least a week between them. $\mathrm{Half}$ of the Ss wore prisms with base left in both versions. half wore them with base right.

\section{Results}

The results of the pointing tests were as follows: For the condition with the arm stationary, there was a shift in the felt location of the fist in the direction of the visual displacement caused by the prism during the exposure period. Only a small, if any, shift occurred for the movement condition. With the results for the two fist locations employed in each test averaged and the results for the two displacement directions combined, the mean difference between the felt fist locations before and after the exposure with arm stationary amounted to $2.4 \mathrm{deg}(\mathrm{p}<.005)$. When the moving arm was observed, the difference amounted to $.5 \pm 1.8 \mathrm{deg}$. The difference between the two means was significant $(\mathrm{p}<.025)$.

\section{DISCUSSION}

Finding larger proprioceptive counteradaptation after viewing a prismatically displaced stationary arm than after observing active movements of the arm under the same conditions furnishes an explanation of Held and Hein's result: they found no visual adaptation under the first condition, but obtained it under the moving condition. In the case of the stationary arm, developing counteradaptation in proprioception seems to delay or to eliminate visual adaptation. Either one of these modifications amounts to process assimilation and can thus compensate for the cue discrepancy caused by the prism during the inspection period. There only remains the question of why little or no proprioceptive counteradaptation develops in the movement condition. This question is easily answered: When $\mathrm{S}$ moves his arm, proprioception is used to control the arm movement. This keeps the relation between proprioceptive cues and the perceived arm locations stable, and only the visual process can change to contribute to process assimilation. When the arm is stationary, proprioception has no such immediate function and is, so to speak, free to change. This explanation also accounts for Held and Hein's finding that passive arm movements as well as immobility failed to produce visual adaptation. Here, proprioceptive control of the arm need not operate either. Inasmuch as we found that inspecting the passive arm leads to a change in the proprioception of its position. the question may be raised as to why Held and Hein did not find evidence for a corresponding change when they tested for the effect of an exposure identical to ours. Unlike our test, theirs employed the same arm in the test that had been inspected in the exposure period. It would seem that a proprioceptive change concerned with the position of the stationary arm will not manifest itself in active movements of that arm.
Held and Hein attributed their result to the presence or absence of reafference, which consisted here of visual stimulation produced by the active arm movements. This stimulation provided visual signals that could be compared with the memory of reafferent signals that had. on earlier occasions, been produced by arm movements that were the result of efferent signals identical to the present ones. These earlier visual signals were, of course, different from those provided by stimulation altered by the prisms. Comparing them would bring about adaptation. This interpretation is supported only by other experiments, where absence of reafference also leads to failure of adaptation to occur. as in the work of Held and Bossom. Our experiments furnish a plausible explanation for Held and Hein's result, Experiment I by demonstrating that visual adaptation and proprioceptive counteradaptation to displaced visual direction can develop concurrently and Experiment II by demonstrating that proprioceptive counteradaptation results from that condition which, for Held and Hein, failed to produce visual adaptation.

That adaptation to displaced visual direction may lead to changes in proprioception is, of course, a well-known fact. It was first demonstrated by Harris (1963) in an experiment in which $\mathrm{S}$ observed for a brief period his pointing arm movements through laterally displacing wedge prisms. The compensatory change in pointing movements that resulted from this training did not transfer to the other arm and proved to be based on a change in proprioception concerned with arm movements. Harris's experiment is in some way analogous to our Experiment $\mathrm{I}$, with his arm movements corresponding to our walking. But Harris failed to obtain visual adaptation: his forward-pointing test had the same result as his pointing-to-a-target test, while we found a significant difference between the results of the forward-walking test and the walking-toward-target test. Under our definition, the effect that Harris obtained has to be regarded as counteradaptation. Why no adaptation in vision was found along with the change in proprioception is not clear at this time.

The process assimilation hypothesis is meant merely to furnish a description of what happens when $S$ is exposed to conditions that may lead to adaptation on the basis of cue alteration. It is not meant to be an ultimate explanation. The exact nature of the process change will probably turn out not to be the same in all cases of adaptation based on cue discrepancy, and hence the concrete causes will differ. In the meantime, the recognition that counteradaptation may develop along with adaptation or instead of it. as a matter of compensation for the discrepancy between paired cues. and that process modification will terminate when process assimilation has become complete has wide application in research on adaptation.

\section{REFERENCES}

Fischer. R.. \& Hill. R. M. Pṣchotropic drus-induced 
transformations of visual space. International Pharmacopsychiatry, 1971, 6, 28-37.

Franklin, S. S.. Ross, H. E., \& Weltman, G. Size-distance invariance in perceptual adaptation. Psychonomic Science, 1970. 21. 229-231.

Gibson. J. J. The perception of the visual world. Boston: Houghton Mifflin, 1950.

Harris, C. S. Adaptation to displaced vision: Visual, motor, or proprioceptive changes? Science, 1963, 140, 812-813.

Hay, J. C., Pick, H. L., Jr., \& Ikeda, K. Visual capture produced by prism spectacles. Psychonomic Science, 1965, 2, 215-216.

Held, R., \& Bossom, J. Neonatal deprivation and adult rearrangement: Complementary techniques for analyzing plastic sensory-motor coordination. Journal of Comparative \& Phy'siological Psychology, 1961, 54, 33-37.

Held, R., \& Hein, A. V. Adaptation of disarranged hand-eye coordination contingent on reafferent stimulation. Perceptual \& Motor Skills, 1958, 8, 87-90.

Rock, I., \& Victor. J. Vision and touch: An experimentally created conflict between two senses. Science, 1964, 143, 594-596.

Shaffer, O.. \& Wallach, H. Adaptation to displaced vision measured with three tests. Psychonomic Science, 1966, 6, 143-144.

Wallach, H. Informational discrepancy as a basis of perceptual adaptation. In S. J. Freedman (Ed.), The neuro-psychology of spatially oriented behavior. Homewood, Ill: Dorsey Press, 1968.

Wallach, H., \& Frey, K. J. On counteradaptation. Perception \& Psychophysics, 1972, 11, 161-165.

Wallach, H., Frey, K. J., \& Bode, K. A. The nature of adaptation in distance perception based on oculomotor cues. Perception \& Psychophysics, 1972, 11, 110-116.

Wallach, H., \& Karsh, E. G. The modification of stereoscopic depth-perception and the kinetic depth-effect. American Journal of Psychology, 1963, 76, 429-435.

Wallach, H., Moore, M. E., \& Davidson, L. Modification of stereoscopic depth-perception. American Journal of Psychology, 1963, 76, 191-204.

Wallach, H., Kravitz, J. H., \& Lindauer, J. A passive condition for rapid adaptation to displaced visual direction. American Journal of Psychology, 1963, 76, 568-578.
Wallach, H., \& Smith, A. Visual and proprioceptive adaptation to altered oculomotor adjustments. Perception \& Psychophysics. $1972,11,413-416$.

\section{NOTES}

1. This statement is not meant as a prediction always verifiable by direct test. See Wallach \& Frey (1972), Note 1.

2 . The term "counteradaptation" has recently been employ'ed by other authors. Fischer and Hill (1971) used it in connection with a study of the effects of certain drugs on thresholds for form distortions. Franklin, Ross, and Weltman (1970) measured adaptation to the effect on distance and size produced by face masks under water. Like us, they used the term "counteradaptation" to refer to nonveridical changes obtained after the adaptation period; but they dealt with size perception, a constancy, and their concept of counteradaptation was not related to process assimilation.

3. An $S$ wearing a laterally displacing prism who, led by $E$, is walking straight ahead will, as a rule, not feel himself to be walking straight ahead but in the direction into which the prism displaces the focus of field expansion. This is in agreement with other cases where visual and proprioceptive inputs are made to conflict with each other; the visual input seems always to dominate proprioceptive experience (Hay, Pick, \& Ikeda, 1965; Rock \& Victor, 1964).

4. This result is slightly larger than one obtained by Shaffer and Wallach (1966) under similar exposure conditions and with an identical test. It is much larger, and significantly so, than one obtained by Held and Bossom (1961) in their Experiment I with a visual forward-direction test that differed from ours in that $S$ varied his own orientation relative to the target.

5. This effect may have been due to a difference in the displacement angle produced by a difference in the location of the prisms relative to the eye.

6. This explanation was proposed by Wallach (1968, p. 225).

(Received for publication October 27, 1972; revision received January 13. 1973.) 